\title{
Qualität, Wirtschaftlichkeit und Innovation in der Pflege: zwei Seniorenzentren im Vergleich
}

Die Überalterung der Gesellschaft, die zu geringe Nachwuchsquote und die viel zu spät gestartete private und öffentliche Vorsorge für die Pflegebedürftigkeit finden ihren Niederschlag im Arbeitsalltag der Sozialund Gesundheitseinrichtungen. Die größte Herausforderung für das Management besteht darin, sowohl Wirtschaftlichkeit als auch materielle und psychosoziale Qualität zu sichern. Das gelingt nur, wenn Planungs- und Steuerungsinstrumente aus der Industrie und kommerziellen Dienstleistungsunternehmen nicht schematisch imitiert, sondern mit Augenmaß adaptiert werden. Zwei Seniorenzentren zeigen, wie das zu schaffen ist.

\section{Friedhart Hegner und Rainer Schadt}

\section{Gesellschaftliche Widersprüche und ihre betrieb- lichen Folgen}

Seit unserer Mitarbeit an den Senioren- und Behindertenplänen zahlreicher Kommunen (z.B. Bielefeld, Braunschweig, Dortmund) in den 80er Jahren stoßen wir immer wieder auf folgende widersprüchliche Entwicklungen:

- Einerseits wachsen die Zahl und der Bevölkerungsanteil der Menschen über 75 Jahre und auch die Zahl der Haushalte, in denen ausschließlich Angehörige der älteren Generation leben, nimmt zu. Andererseits schrumpfen die Zahl und der Anteil derjenigen Senioren, die durch eigenen Nachwuchs zumindest die Möglichkeit eröffnet haben, dass Ihnen bei Krankheit oder Pflegebedürftigkeit nicht-professionelle Hilfe zuteil wird (Kaufmann 2005). Zusätzlich schwindet das Potenzial nicht-professioneller Hilfe durch zahlreiche andere Faktoren wie z.B. Erwerbstätigkeit von Frauen und Männern, räumliche Trennung der Generationen aufgrund beruflicher Mobilität (Hegner/Lakemann 1989, Kaufmann 1997).

- Nächstes Dilemma: Einerseits stößt die Ausweitung professioneller Hilfe- und Pflegeangebote an finanzielle Grenzen, weil weder die privaten noch die öffentlichen Haushalte (inkl. Sozialversicherungsträger) bereits in

Dr. Friedhart Hegner, Geschäftsführender Gesellschafter der ISMV Dr. Hegner \& Partner GmbH (Institut für Sozialplanung, Management und Verwaltung) Berlin und Bielefeld; Dipl.-Kfm. Rainer Schadt, Projektleiter im Büro Berlin der ISMV GmbH. den 50er Jahren damit begonnen haben, Rücklagen für das - voraussehbare - wachsende Pflegerisiko zu bilden (Kaufmann 1997). Andererseits hat Heddy Neumeister bereits 1962 unter dem prägnanten Titel „Organisierte Menschlichkeit? Grenzen des Sozialen Fortschritts“ klargestellt, dass professionelle Hilfe- und Pflegeangebote nicht nur an budgetäre Grenzen stoßen, sondern vor allem auch an psychosoziale (Hegner 1996). Behandlungs-, Körper- und Haushaltspflege können professionell bzw. ablauftechnisch bei entsprechenden materiellen Ressourcen durchaus auf akzeptablem Qualitätsniveau organisiert werden, was hinsichtlich individueller Zuwendung, sozialer Kommunikation und aktivierender Hilfe bzw. Betreuung sehr viel schwieriger ist (so bereits die empirischen Daten bei Dahme u.a. 1980; siehe auch Evers/Olk 1996).

Diese widersprüchlichen gesamtgesellschaftlichen Entwicklungen prägen das Alltagshandeln auf der MikroEbene einzelner Wohn- und Pflegeeinrichtungen, aber auch bei der ambulanten Pflege. Wie der Spagat zwischen knappen Ressourcen und hohen Anforderungen an die Pflegequalität in der Alltagspraxis bewältigt wird, zeigen die beiden folgenden Fallstudien.

Vor Darstellung der Organisationsverbesserungen und Steuerungsinstrumente einige Daten zum Vergleich beider Einrichtungen und zur Projektdurchführung.

\section{Merkmale der beiden Einrichtungen: Gemeinsamkeiten und Unterschiede}

Das gemeinsame Ausgangsproblem beider Einrichtungen - zugleich der Anlass für den Wunsch nach einer 
Tabelle 1: Eckdaten zu den beiden Einrichtungen

\begin{tabular}{|c|c|c|}
\hline & Einrichtung Nordwind & Einrichtung Südwind \\
\hline Zahl der Plätze & $\begin{array}{l}190 \text { vollstationäre Plätze } \\
7 \text { Plätze für Kurzzeitpflege }\end{array}$ & $\begin{array}{l}115 \text { vollstationäre Plätze } \\
17 \text { Tagespflegeplätze }\end{array}$ \\
\hline Zahl der Mitarbeiter & $\begin{array}{l}88 \text { Gesamt } \\
65 \text { direkt in der Pflege }\end{array}$ & $\begin{array}{l}56 \text { Gesamt } \\
36 \text { direkt in der Pflege }\end{array}$ \\
\hline $\begin{array}{l}\text { Quote } \\
\text { Teilzeitbeschäftigte }\end{array}$ & $13 \%$ & $70 \%$ \\
\hline Quote Fachpflegekräfte & $52 \%$ & $53 \%$ \\
\hline Essensversorgung & $\begin{array}{l}\text { Im Hause, Anlieferung der } \\
\text { Warmgerichte von außen }\end{array}$ & Küche und Bevorratung im Hause \\
\hline $\begin{array}{l}\text { Tagestrukturierung/thera- } \\
\text { peutische Angebote }\end{array}$ & $\begin{array}{l}\text { Altersgerechte Gymnastik, Ausflüge } \\
\text { „mit Programm“ (z.B. Museum, } \\
\text { Theater), Spiel- und Tanznachmittage, } \\
\text { Chorsingen }\end{array}$ & $\begin{array}{l}\text { ADL-Selbsthilfetraining (Aktivitäten des } \\
\text { täglichen Lebens), Altersgerechte } \\
\text { Gymnastik, Kreatives Gestalten, } \\
\text { „Tanzen hält fit“ - Tanznachmittage, } \\
\text { Denksport und Spiele }\end{array}$ \\
\hline \multicolumn{3}{|c|}{$\begin{array}{l}\text { Das praxisbezogene empirische Projekt umfasste über einen Zeitraum von jeweils knapp zwei Jahren gemäß } \\
\text { der Fallstudien-Methodik folgende Schritte: Analyse schriftlicher Dokumente und statistischer Daten zu Budget- } \\
\text { und Qualitätsthemen; strukturierte mündliche Interviews mit Pflege-, Verwaltungs- und Hauswirtschaftskräften } \\
\text { sowie den jeweiligen Leitungspersonen; Bildung eines Projektteams aus Mitarbeitern und Leitungskräften aller } \\
\text { Funktionsbereiche für jede der beiden Einrichtungen; Beratung bei der Entwicklung und Einführung von } \\
\text { Steuerungsinstrumenten und Organisationsverbesserungen. }\end{array}$} \\
\hline
\end{tabular}

Quelle: ISMV Dr. Hegner \& Partner $\mathrm{GmbH}$

Organisationsberatung - lässt sich am besten mit folgenden Zitaten aus den Eingangsgesprächen verdeutlichen:

- „Wie alle Einrichtungen mit einem hohen Anteil Pflegebedürftiger sind wir ständig in der Sorge, einen positiven Jahresabschluss zu erreichen. Noch mehr Sorge bereiten uns allerdings die Klagen von Angehörigen darüber, dass unser Personal lieblos mit den Bewohnern umgehe. Das schadet unserem Ruf und gefährdet die Auslastung." (Sprecher des Stiftungsrats der Senioreneinrichtung Nordwind, so unsere anonymisierte Bezeichnung für die dortige Gruppe von drei Heimen in Norddeutschland)

- „Die Arbeitsbelastung ist enorm und steigt weiter. Mehr Personal können wir uns nicht leisten, weil sonst die Bilanz nicht stimmt. Bei Rundgängen stelle ich immer wieder das Gleiche wie manche unserer Besucher (Angehörige) fest: Rein pflegetechnisch gesehen haben wir zwar immer noch zu viele Risiken, aber im Großen und Ganzen klappt es einwandfrei. Große Sorge be- reitet mir, dass unsere Bewohner oft wortlos - um nicht zu sagen lieblos - gebettet, im Rollstuhl geschoben oder mit Essen versorgt werden." (Heimleitung der Senioreneinrichtung Südwind, einem der großen Wohlfahrtsverbände in Süddeutschland angeschlossen)

In den Interviews mit den Mitarbeitern und Führungskräften werden ähnliche Themen, allerdings mit anderen Akzentsetzungen angesprochen:

- Angesichts des großen Zeitdrucks sei man froh, bei der Grund- und Behandlungspflege wenigstens die geltenden Vorschriften und fachlichen Standards einzuhalten, um möglichst keine Risiken zu erzeugen. Allerdings hänge der Zeitdruck auch davon ab, wie geschickt jede(r) seine Arbeit organisiere und wie geübt man bei den einzelnen Handgriffen sei.

- Ob man den Bewohnern Zuwendung und ein persönliches Wort gebe, hänge keineswegs nur vom Zeitdruck ab. Einerseits finde man auch bei Kollegen mit viel Erfahrung und Geschick, also mit ausreichend Zeit für persönliche Pausen (z.B. Kollegengespräche), völliges 
Desinteresse am psychischen Wohlergehen der Bewohner. Andererseits gebe es Kollegen und Kolleginnen, bei denen der Zeitdruck größer sei und dennoch immer wieder ein Gespräch mit den Bewohnern geführt werde.

- Ähnlich sei es bei der Aktivierung der Bewohner. Manche Kollegen und Kolleginnen hätten nur das Bestreben, möglichst wenig Zeit bei der Darreichung der Mahlzeiten, bei Begleitgängen oder bei der Körperpflege zu verlieren. Deshalb würden die Bewohner so wenig wie möglich aktiv einbezogen. Demgegenüber treffe man bei anderen Pflegekräften ständig auf das Bemühen, die Bewohner anzuleiten, bestimmte Alltagsverrichtungen eigenständig zu erledigen.

Ähnlich geteilt sind die Auffassungen hinsichtlich der Umsetzung von Innovationen. Knapp zwei Fünftel der Mitarbeiter und Führungskräfte halten die PC-gestützte Pflegedokumentation (inkl. Qualitätskontrolle) - also ein Beispiel für eine Organisationsveränderung - für ebenso unnötig aufwändig wie beispielsweise Maßnahmen zu einer flexibleren Gestaltung der Essens- und Ruhezeiten oder zur Aktivierung der Bewohner, also fachliche Neuerungen bei den Dienstleistungen.

Hinsichtlich der Akzentsetzungen bei innovativen Dienstleistungsangeboten (siehe allgemein Landenberger/Münch 2001) unterscheiden sich die beiden Einrichtungen:

- Einrichtung Nordwind legt besonderen Wert auf die fachliche Intensivierung der Betreuung Demenzkranker und die Erweiterung der Platzzahlen in der Gerontopsychiatrie (vor allem zwecks frühzeitiger Maßnahmen bei Demenzerkrankungen). Daneben wird der Aufbau einer Wohneinrichtung für rüstige Senioren betrieben.

- Einrichtung Südwind sieht den Innovationsschwerpunkt bei der Tagespflege und bei Kurzzeitpflegeplätzen, also bei Maßnahmen zur Unterstützung der Angehörigen von Senioren. Die Vorbereitungen für gerontopsychiatrische Angebote laufen wegen des

Fachkräftemangels mühsam.

Neben budgetären und fachlichen Gründen führen die Heimleitungen beider Einrichtungen als weiteres Motiv für die genannten Innovationen an: Da die Betreuung hochgradig Pflegebedürftiger eine auf Dauer sowohl körperlich als auch psychisch belastende Aufgabe sei, werde es immer schwieriger, dafür geeignetes Personal zu finden, und - vor allem - dauerhaft an die Einrichtung zu binden. Deshalb müsse man nach Möglichkeiten suchen, um das Personal phasenweise mit körperlich und psychisch weniger belastenden Aufgaben zu betrauen (z.B. bei rüstigen Senioren). Das gelinge allerdings nur bei den Mitarbeitern, die bereit seien, dafür auch zusätzliche Qualifizierungsanstrengungen auf sich zu nehmen (z.B. ausgeprägt aktivierende und variable Betreuung in der Tagespflege).

\section{Verbesserung des Budgetmanagements}

Alle Leitungskräfte beider Einrichtungen waren und sind bestrebt, die Wirtschaftlichkeit zu verbessern. Da es sich um gemeinnützige Einrichtungen handelt, sollen die angestrebten Überschüsse ausschließlich dazu dienen, notwendige Verbesserungen und Innovationen sowohl bei der organisatorischen und räumlich-technischen Infrastruktur als auch bei den Dienstleistungsangeboten zu finanzieren. Obwohl man - so beide Heimleitungen - den Mitarbeitern immer wieder gesagt habe, dass Überschüsse für Maßnahmen sowohl der Bewohner als auch der Belegschaft verwendet würden, sei das Interesse an einer Verbesserung von Effektivität und Effizienz überwiegend gering. Wie lässt sich das verändern? Beide Einrichtungen haben sich für folgendes Vorgehen entschieden, bevor eine handlungsleitende Kennziffer als Steuerungsinstrument entwickelt wurde (siehe generell Hegner 2000):

Zunächst kommt es darauf an, abstrakte ökonomische Kategorien in eine für das Alltagshandeln bedeutsame Form zu übersetzen. Das beginnt mit der - für Pflegekräfte zunächst ungewöhnlichen - Frage nach dem wichtigsten Produktionsfaktor. Das ist im Sozial- und Gesundheitsbereich, wenn man von technologieintensiven Medizinbereichen absieht, der Faktor Arbeitskraft. Also müssen die verbrauchten Arbeitsstunden sowie die Art der Arbeitszeit- und Urlaubsgestaltung besonders beachtet werden.

Botschaft an die Mitarbeiter in Info-Runden mit anschließender Verteilung von Handzetteln/Flyern mit folgenden Themen:

1. Durch geschickte Absprachen in Teams oder sogar in größeren Bereichen zum einen Beginn und Ende sowie zum Zweiten die Länge der Arbeitszeiten pro Tag und Woche sowohl an den Arbeitsanfall als auch an Personalausfälle durch Krankheit, Urlaub usw. anpassen.

2. Auf diese Weise den Anteil der produktiv eingesetzten Arbeitsstunden steigern. Zugleich die Aufwendungen für zuschlagspflichtige Mehrarbeit senken.

3. Ähnliches bei der Urlaubsplanung beachten. Wenn es den Mitarbeitern gelingt, durch geschickte Absprachen gemäß den familiären Besonderheiten (z.B. Schulferien von Kindern) die individuellen Jahresurlaube gestaffelt zu legen, verringern sich die Ausgaben für Zeitarbeitskräfte und Überstunden in den Sommermonaten sowie um die Jahreswende.

Weitere Botschaft an die Mitarbeiter: Im Dienstleistungsbereich spielt der Wirkungsgrad (Effektivität) des Arbeitskrafteinsatzes eine große Rolle. Was bedeutet das für den Alltag? Vom Wirkungsgrad werden folgende Faktoren maßgeblich beeinflusst, was von den Pflegekräften erst nach und nach akzeptiert wurde:

- Auslastung der Plätze und Umsatz, wobei beides davon abhängt, welche Position die Einrichtung im regionalen Wettbewerb um potenzielle Bewohner hat, was 
wiederum von der Dienstleistungsqualität und von den Preisen abhängt.

- Preisniveau, also Höhe der von den Bewohnern oder ihren Angehörigen zu zahlenden Gebühren, wobei das Preisniveau oberhalb des Kostenniveaus liegen muss, um wirtschaftlich zu überleben. Da das Preisniveau vom regionalen Wettbewerb abhängt, kommt es für die Mitarbeiter vor allem darauf an, das Kostenniveau so niedrig wie möglich zu halten.

- Die Höhe des Kostenniveaus wird vorrangig davon beeinflusst, wie produktiv die Mitarbeiter ihre Arbeitszeit nutzen (siehe oben) und wie sorgfältig sie mit den ihnen anvertrauten Sachgütern umgehen.

- Die Aufwendungen für Sachgüter werden von den Mitarbeitern in zweierlei Hinsicht beeinflusst: zum einen beim Umgang mit langlebigen Gebrauchsgütern, also beispielsweise bei der Bedienung und Bewegung von Pflegebetten, Rollstühlen, Pflegewagen, Mobiliar usw.; zum Zweiten beim Umgang mit Verbrauchsmaterialien, also beispielsweise mit Verbandsmaterial, Inkontinenzmitteln, Stützstrümpfen, Nahrungsmitteln, Getränken usw.

- Wer glaubt, das alles seien Trivialitäten, kennt den betrieblichen Alltag nicht. Es erfordert stunden- oder sogar tagelange Diskussionen, bis die Mitarbeiter davon überzeugt sind, dass sie die zuvor genannten Kostengrößen tatsächlich beeinflussen können. Dabei wird der ,schwarze Peter' immer wieder zwischen den Führungskräften und Mitarbeitern hin und her geschoben. Am Ende der Überzeugungsarbeit steht der Konsens für eine der beiden folgenden Formeln:

\section{Abbildung 1: Kennziffern zum Budgetmanagement}

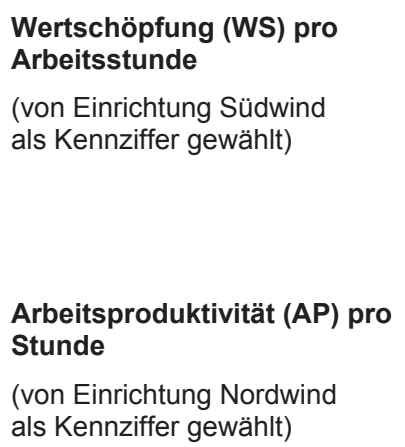

Arbeitsproduktivität (AP) pro Stunde

(von Einrichtung Nordwind als Kennziffer gewählt)

WS p. H. =

bezahlte Arbeitsstunden (inkl. Ausfallzeiten wie
Umsatz- beeinflussbare Kosten Urlaub usw.)

wicklung bei der Wertschöpfung bzw. Produktivität pro Stunde.

- Info-Runden wenige Tage nach dem Aushang. Dabei informiert die Geschäfts-bzw. Heimleitung zunächst die Bereichsleitungskräfte und lädt zur Erörterung der positiven und negativen Entwicklungen ein. Anschließend diskutieren die Bereichsleitungskräfte mit ihren Mitarbeitern im kleinen Kreis darüber, warum sich einzelne Werte verbessert oder verschlechtert haben und was vorrangig zu tun ist.

Das doppelgleisige Vorgehen wurde gewählt, weil sich bereits wenige Monate nach den ersten Datenaushängen zeigte, dass die alleinige schriftliche Information wenig bewirkt. Jedoch verdoppelt sich die Informationswirksamkeit, wenn in einer ca. 45-60minütigen mündlichen Erörterung geprüft wird, durch welche konkreten Maßnahmen sich gute Datenwerte stabilisieren und schlechte Datenwerte verbessern lassen. Die aufgewendete Zeit zahlt sich durch Zeiteinsparungen aufgrund der vereinbarten Maßnahmen aus.

\section{Veränderungen beim Qualitätsmanagement}

Der notwendige Aufwand für die Einführung der Daten zur Wirtschaftlichkeit veranlasste die Leitungsgremien beider Einrichtungen zu der Frage, ob man nicht die Bereitstellung der Qualitätsdaten zeitlich verschieben könne. Vertretbar ist ein solches stufenweises Vorgehen dann, wenn die Veröffentlichung der Qualitätsdaten maximal 3 Monate nach der Erstveröffentlichung der Budgetdaten erfolgt. Bei einem größeren zeitlichen Abstand haben wir die bittere Erfahrung gemacht, dass sich alle Beteiligten auf die Optimierung der Wirtschaftlichkeit konzentrieren und gleichzeitig die Qualitätsrisiken steigen (z.B. dokumentiert durch Beschwerden von Angehörigen oder des Medizinischen Diensts der Krankenkassen).

Statt einer zeitlichen Verschiebung haben sich beide Einrichtungen für eine Aufspaltung und stufenweise Abarbeitung des Qualitätsthemas entschieden (siehe allgemein Hegner 2000):

- Vorrang für die als matetatsächlich geleistete Arbeitsstunden (abzügl Ausfallzeiten wie Krankheit, Urlaub usw.)
AP p. $H .=$

Umsatz- beeinflussbare Kosten

Quelle: ISMV Dr. Hegner \& Partner $\mathrm{GmbH}$

Je nach Handhabung der verfügbaren Software im kaufmännischen Bereich der Einrichtungen werden die obigen Daten teils monatlich und teils pro Quartal zur Verfügung gestellt. Das geschieht auf zweierlei Weise, um die Mitarbeiter nicht nur zu informieren, sondern auch zu aktivieren:

- Aushang eines grafisch gut aufbereiteten Diagramms mit Entwicklung der Umsätze, der wichtigsten Kostenarten sowie des Stundenverbrauchs, ergänzt um die Ent- riell bzw. instrumentell bezeichneten Qualitätsthemen. Dazu zählen die überwiegend objektiv messbaren Abweichungen zwischen Soll- und Ist-Qualität bei Maßnahmen der Grund- und Behandlungspflege sowie bei der Zubereitung und Darreichung von Nahrungsmitteln.

- Verschiebung der Kontrolle immaterieller bzw. expressiv-psychosozialer Qualitätsaspekte um rund ein Jahr; also vorerst keine Erfassung von Soll-Ist-Abwei- 
chungen hinsichtlich Art der Gesprächsführung zwischen Bewohnern und Mitarbeitern, Grad der Ermutigung zwecks Aktivierung bzw. Mobilisierung bei Alltagsverrichtungen, Motivierung zur Teilnahme an tagesstrukturierenden oder/und therapeutischen Angeboten.

Den Leitungsgremien ist diese Prioritätensetzung nicht leicht gefallen. Angesichts der vielfachen Klagen über "lieblose Behandlung“ hätten sie dieses Thema gerne so früh wie möglich angepackt. Wer jedoch schon einmal versucht hat, Kennziffern für Wirtschaftlichkeit und für die materielle Qualität in Sozial- und Gesundheitseinrichtungen einzuführen und(!) handlungswirksam zu machen, weiß Folgendes: Nur ein schrittweises Vorgehen bewahrt davor, viele Neuerungen ohne nachhaltige Effekte zu proklamieren, statt wenigstens einige davon nachhaltig zu realisieren und zu stabilisieren. Deshalb konzentrieren wir uns an dieser Stelle auf die Kontrolle der materiellen bzw. instrumentellen Qualität, obwohl beide Einrichtungen inzwischen - nach Ablauf von mehr als 2 Jahren - mit der immateriellen Qualitätsmessung begonnen haben.

Auf den ersten Blick erscheint die Erarbeitung eines Prüfbogens für die materielle Qualität wegen der zahlreich vorliegenden Formulare einfach und selbstverständlich. Auch die Fachleute in den beiden Einrichtungen waren sich relativ rasch einig, auf welche Aspekte man achten muss, um Qualitätsrisiken zu vermeiden. Viel schwieriger war es, mit den Mitarbeitern und Mitarbeitervertretungen/Betriebsräten einen Konsens bei folgender Frage herzustellen: Wie kann bei jeder Kategorie ein objektivierter Soll-Ist-Abgleich erfolgen, um subjektiv gefärbte Verzerrungen bei der Qualitätsbeurteilung zu vermeiden? Bei der Suche nach Kategorien, die dem in der Frage enthaltenen Anspruch gerecht werden, musste in beiden Einrichtungen auf viele der gängigen und oft hochgestochenen Formulierungen aus Qualitätshandbüchern verzichtet werden. Stattdessen haben sich alle Beteiligten auf - kleinlich erscheinende - handwerkliche Kategorien verständigt.

Der folgende Auszug aus dem Q-Erfassungsbogen enthält vor allem jene Prüfkriterien, die besonders wichtig sind, um Pflegerisiken zu vermeiden. (Tabelle 2)

Der Qualitätsprüfbogen wird jedes Jahr einer Revision unterzogen. Dabei werden zum einen noch schwammige Kategorien weiter konkretisiert und zum Zweiten fehlende Kategorien ergänzt, wobei die früheren Erfahrungen bei Anwendung des Bogens nützen. Ebenso wie bei der Budgetkennziffer werden auch bei der Qualitätskennziffer - auf Basis der skizzierten Ansprüche an die praktische Wirksamkeit - die Erfahrungen der Mitarbeiter durch zwei Maßnahmen aktiviert und genutzt:

- Aushang der jeweils aktuellen Kennziffernwerte, wobei zum Vergleich die früheren Werte ebenfalls sichtbar gemacht werden.

- Erörterung der Gründe bzw. Ursachen für die jeweils erreichten Qualitätswerte, um durch darauf gerichtete
Maßnahmen zum einen verbesserte Werte stabilisieren und zum anderen schlechte Werte verbessern zu können.

Die Erfahrungen aus den zurückliegenden Jahren zeigen, dass dieses doppelgleisige Vorgehen die Sensibilität der Mitarbeiter für Qualitätsfragen eher verstärkt als ständige Ermahnungen, also verbale normative Hinweise auf vorliegende Qualitätsrichtlinien (z.B. im Qualitätshandbuch). Damit soll keineswegs gesagt werden, dass ein Qualitätshandbuch verzichtbar ist.

\section{Verbesserte Prozess- und Wirkungsqualität: nur dauerhaft bei passgerechter Strukturqualität}

Die zuvor skizzierten Maßnahmen tragen ausschließlich zur Verbesserung der Prozessqualität bei. Sie gelingen in der Regel nur, wenn die Leitungsgremien ergänzend Verbesserungen der Strukturqualität auf den Weg bringen (Hegner 2000, Landenberger/Münch 2001). Das hat außerdem folgenden Vorteil: Die Zustimmung der Belegschaft und der Mitarbeitervertretung ist leichter zu gewinnen, wenn parallel Defizite bei der Programmstruktur (z.B. Widersprüche in der Angebotspalette) sowie bei der Organisationsstruktur (z.B. unklare oder zu lange Entscheidungswege) beseitigt werden. Das ist besonders dann wichtig, wenn die Wettbewerbsfähigkeit durch parallele Korrekturen beim Budget- und Qualitätsmanagement gestärkt werden soll. Dazu einige Beispiele aus den beiden Einrichtungen:

a) Klare Kompetenzabgrenzungen und ein gutes Zusammenspiel zwischen den verschiedenen Leitungsgremien bilden die Mindestvoraussetzung für eine wirksame Steigerung sowohl der Wirtschaftlichkeit als auch der Qualität. Hier hat die Einrichtung Nordwind deutliche Startvorteile gegenüber der Einrichtung Südwind. Stiftungsrat, Vorstand und Heimleitung bei Nordwind hatten zwar anfangs unterschiedliche Auffassungen über die Geschwindigkeit der notwendigen Verbesserungsmaßnahmen, konnten sich jedoch nach kurzer und intensiver Diskussion auf einen gemeinsamen inhaltlichen und terminlichen Nenner verständigen. Demgegenüber traten bei Südwind Verzögerungen und Reibungsverluste auf, weil Gesellschafterversammlung, Geschäftsführung und Heimleitung sich über lange Zeit hinweg nur partiell auf die Zielsetzungen und Schritte des Veränderungsprozesses verständigen konnten.

b) Die Verstärkung der Selbstkontrolle der Mitarbeiter durch Kennziffern und diesbezügliche Info-Runden gelingt nachhaltig nur dann, wenn durch technologische Hilfsmittel die Alltagsarbeit erleichtert wird (siehe allgemein Fraunhofer 2006). Im Falle von Nordwind war die zügige Einführung geeigneter Soft- und Hardware zur Generierung und Pflege der Budget- und Qualitätsdaten ein wichtiger Beitrag, um den Mitarbeitern glaubhaft zu machen, dass alles getan wird, um die Arbeit von der technischen Seite her zu erleichtern. In die gleiche Richtung wirken die von Anfang an zugesagten und realisierten Verbesserungen bei Pflegegerä- 
Tabelle 2: Auszug aus dem Prüfbogen für Qualität für die materielle Pflegequalität

\begin{tabular}{|c|c|c|c|c|c|c|}
\hline I. Qualität des Dokumentierens & positiv & $\begin{array}{l}\text { ansatz- } \\
\text { weise }\end{array}$ & $\begin{array}{c}\text { (fast) } \\
\text { negativ }\end{array}$ & $\begin{array}{c}\text { Bemerkungen/ } \\
\text { Maßnahme }\end{array}$ & Termin & Wer \\
\hline \multicolumn{7}{|l|}{ I.1 Eintragung im Stammblatt vollständig? } \\
\hline \multicolumn{7}{|l|}{ I.2 Anamnese vollständig aktualisiert? } \\
\hline \multicolumn{7}{|l|}{$\begin{array}{l}\text { I.3 Berichteblatt aussagefähig und nach- } \\
\text { vollziehbar? }\end{array}$} \\
\hline \multicolumn{7}{|l|}{$\begin{array}{l}\text { I.4 Anwendung fachlich richtiger Bezeich- } \\
\text { nungen u. Abkürzungen im Berichteblatt? }\end{array}$} \\
\hline \multicolumn{7}{|l|}{$\begin{array}{l}\text { I.5 Durchführungsnachweise Grund- und } \\
\text { Behandlungspflege sowie Therapie/Akti- } \\
\text { vierende Betreuung regelmäßig, zeitnah } \\
\text { und nachvollziehbar? }\end{array}$} \\
\hline \multicolumn{7}{|l|}{ II. Qualität der Pflegeplanung (Auszug) } \\
\hline \multicolumn{7}{|l|}{$\begin{array}{l}\text { II.1 Werden alle Ressourcen und Defizite } \\
\text { der Bewohner entsprechend des Pflege- } \\
\text { konzeptes (AEDL) erfasst? }\end{array}$} \\
\hline \multicolumn{7}{|l|}{$\begin{array}{l}\text { II.2 Werden die Pflegeziele unter Berück- } \\
\text { sichtigung der Bedürfnisse, Ressourcen } \\
\text { und Defizite der Bewohner klar und präzise } \\
\text { formuliert? }\end{array}$} \\
\hline \multicolumn{7}{|l|}{$\begin{array}{l}\text { II.3 Erfolgt die Zuordnung von Pflegemaß- } \\
\text { nahmen zu den Pflegezielen gut begrün- } \\
\text { det? }\end{array}$} \\
\hline \multicolumn{7}{|l|}{$\begin{array}{l}\text { II.4 Wurden alle } 10 \text { bis } 12 \text { Wochen positive } \\
\text { und/oder negative (Zwischen-)Ergebnisse } \\
\text { dokumentiert? }\end{array}$} \\
\hline \multicolumn{7}{|c|}{ III. Qualität der Durchführung von Pflegemaßnahmen (Auszug) } \\
\hline \multicolumn{7}{|l|}{$\begin{array}{l}\text { III.1 Wird der Bewohner auf der Basis der } \\
\text { Pflegeplanung bei der Durchführung von } \\
\text { Maßnahmen aktiv einbezogen, d.h. zur } \\
\text { Mitwirkung ermutigt und bei der Ausfüh- } \\
\text { rung unterstützt? }\end{array}$} \\
\hline $\begin{array}{l}\text { III. } 2 \text { Werden andere Beteiligte entspre- } \\
\text { chend der Pflegeplanung in den Pflege- } \\
\text { prozess (z.B. Angehörige, Betreuer, Arzt, } \\
\text { Mitarbeiter Therapie) einbezogen? }\end{array}$ & & & & & & \\
\hline
\end{tabular}

Quelle: ISMV Dr. Hegner \& Partner $\mathrm{GmbH}$

ten, Mobilitätshilfen, Kommunikationsinstrumenten usw. Diese Verbesserung der technischen Rahmenbedingungen erfolgte bei Südwind langsamer und in geringerem Umfang, weil aufgrund der Uneinigkeit bei den Leitungsgremien die örtlichen Einrichtungen vieles in Eigenregie - neben der Alltagsarbeit - erledigen mussten.

c)Unverzichtbar sind auch personalstrukturelle Begleitmaßnahmen. Hier hat Nordwind folgende Schritte reali- siert: 1. Auf Basis eines Leitfadens erfolgende Personalbeurteilung, um diejenigen Mitarbeiter, die den Budgetund Qualitätsmaßnahmen aufgeschlossen gegenüberstehen, von den Zauderern und Widerspenstigen zu unterscheiden; 2. Verstärkung der Trainingsmaßnahmen für die Zauderer, also über die allgemeinen Basistrainingsmaßnahmen hinaus; 3. gezielte Stellenausschreibungen zwecks Verstärkung der Belegschaft um Fachkräfte, die einer Selbstkontrolle durch Budget- und Qualitätskennziffern aufgeschlossen gegenüberstehen; 4 . keinerlei 
Widerstände gegen Eigenkündigungen jener Mitarbeiter, die sich durch die veränderten Rahmenbedingungen unter Druck gesetzt fühlen. - Leider konnte die Heimleitung von Südwind nicht ähnlich konsequent vorgehen, weil ihr die volle Unterstützung aller übergeordneten Leitungsgremien fehlte.

Was resultiert aus den unterschiedlichen Rahmenbedingungen für die budget- und qualitätsbezogenen Resultate der Verbesserungsmaßnahmen? Besonders bemerkenswert ist, dass Südwind trotz der ungünstigeren Rahmenbedingungen nach einer Anlaufphase in zwei aufeinander folgenden Jahren sowohl die Wertsteigerung pro Stunde als auch die materielle Qualität spürbar verbessern konnte. Noch deutlicher war allerdings die positive Entwicklung bei den Budget- und Qualitätskennziffern im Falle Nordwind. Aufgrund der nachweisbaren Erfolge konnte Nordwind in den Pflegesatzverhandlungen - allerdings erst nach insgesamt mehr als zwölfstündigen Gesprächen - eine über dem durchschnittlichen Anstieg der letzten Jahre liegende Steigerung der Pflegesätze erreichen. Das wiederum hat die Motivation der Führungskräfte und Mitarbeiter zur Realisierung weiterer Verbesserungen gestärkt.

Wieso konnte bei Südwind trotz der ungünstigeren Rahmenbedingungen dennoch über zwei Jahre hinweg eine Verbesserung realisiert werden? Die Gespräche mit den Führungskräften und Mitarbeitern dokumentieren, dass einer der wichtigsten Motivatoren darin bestand, sowohl die Budgetkennziffer als auch die Qualitätskennziffer völlig zu durchschauen. Neben der diesbezüglichen Überzeugungsarbeit hat als weiterer Motivator die Transparenz der Zusammenhänge zwischen Budget- und Qualitätsmanagement positiv gewirkt.

\section{Stabilisierung positiver Ergebnisse durch leistungsbezogene Entgeltbestandteile}

Wieso haben diese positiven Schubkräfte nach Ablauf von rund zwei Jahren kontinuierlich an Wirksamkeit verloren? Dazu drei Antworten aus Sicht der Führungskräfte und Mitarbeiter: 1 . wegen der in den ersten Jahren erreichten starken Verbesserungen, die den Spielraum für weitere Steigerungen eingeengt haben; 2. weil die Uneinigkeit der Leitungsgremien für die Belegschaft immer deutlicher erkennbar wurde und somit die Fraktion der Widerspenstigen Rückenwind bekam; 3. wegen der Nichtberücksichtigung des Wunsches der besonders tatkräftigen Mitarbeiter, eine finanzielle Vergütung für die überdurchschnittlichen Leistungen zu erhalten. Das hatte die Heimleitung angestrebt, war jedoch von den übergeordneten Gremien und der Mitarbeitervertretung verweigert worden.

Hier hat Nordwind einen anderen Weg eingeschlagen (siehe allgemein Bretschneider u.a. 1999). Von allen Leitungsgremien wurde seit Start der Veränderungen immer wieder betont, dass man leistungsbezogene Entgeltkomponenten einführen wolle, sobald die Kennziffern und die Info-Runden stabilisiert seien. Durch diese konsequente Haltung fühlen sich die leistungsstarken Mitarbeiter (,Zugpferde') in ihrem Verbesserungsstreben bestärkt, während die ,Mitläufer' immer wieder angespornt werden, wenigstens kleine Verbesserungen bei der Wirtschaftlichkeit oder/und Qualität zu bewirken. Die Verhandlungen über ein diesbezügliches Entgeltsystem waren nicht einfach und zogen sich über mehr als ein Jahr hin, konnten jedoch inzwischen durch eine Kollektivregelung abgeschlossen werden.

Bei Nordwind wird die Wirksamkeit der leistungsbezogenen Komponenten durch folgende Kombination von Steuerungsinstrumenten verstärkt: zum einen die genannten Kennziffern und Info-Runden; zum Zweiten eine systematische Verhaltensbeurteilung, um mit Hilfe objektivierter Kriterien die individuellen Beiträge zu den kollektiven Ergebnisverbesserungen honorieren zu können. - Bei Südwind wird die Wiederbelebung des anfänglichen Reformeifers der Belegschaftsmehrheit durch eine andersartige Maßnahmekombination angestrebt:

- zum einen Verstärkung der zwischenzeitlich vernachlässigten Trainingsmaßnahmen, um sowohl neu hinzugekommene Führungskräfte und Mitarbeiter als auch Zauderer davon zu überzeugen, wie wichtig kombiniertes Budget- und Qualitätsmanagement für eine starke Position im Wettbewerb ist;

- zum Zweiten durch Einführung einer systematischen Verhaltensbeurteilung, um durch Veröffentlichung der Punktwerte wenigstens sichtbar zu machen, welche Unterschiede zwischen den ,Zugpferden' und ,Mitläufern' bestehen.

Eine solche Kombination mehrerer immaterieller Anreize ist durchaus sinnvoll. Sie erhöht auch bei Anwendung leistungsbezogener Entgeltkomponenten, also materieller Anreize, die Wirksamkeit der Verbesserungsmaßnahmen, wie das Beispiel Nordwind zeigt. Alle Anreize können allerdings die angestrebten positiven Effekte nur dann erreichen, wenn parallel die strukturellen Rahmenbedingungen verbessert werden. Motto: Prozess- und Wirkungsqualität von personalen Dienstleistungen lassen sich dauerhaft nur verbunden mit einer verbesserten Strukturqualität steigern.

\section{Zusammenfassung}

Demographische Verwerfungen sowie Risikoverschiebungen auf Seiten der privaten und öffentlichen Haushalte finden ihren Niederschlag im Alltagsleben der Menschen. Das gilt für Hilfe- und Pflegebedürftige ebenso wie für die professionellen Helfer. In den kombinierten Wohn- und Pflegeeinrichtungen für Senioren geht es um den permanent schwierigen Balanceakt zwischen Budgeterfordernissen sowie materiellen und immateriellen Qualitätsanforderungen, verbunden mit der Notwendigkeit, fachlich anerkannte Innovationen bei den Dienstleistungen zu realisieren. Mit Hilfe welcher Steuerungsinstrumente dieser ständige Balanceakt in der Alltagspraxis bewältigt werden kann, wird am Beispiel von zwei 
Seniorenzentren illustriert. In beiden Fällen trägt die Kombination von Budget- und Qualitätskennziffern zusammen mit Informationsmaßnahmen dazu bei, zum einen die Wirtschaftlichkeit zu erhöhen sowie zum Zweiten ein hohes Qualitätsniveau zu sichern.

\section{Literaturverzeichnis}

Bretschneider, M./F. Hegner/ R. Kleibs 1999: Was kommt nach dem BAT? Grundzüge eines Entgeltsystems mit Steuerungseffekten im Wohlfahrtsbereich. In: Arbeit und Sozialpolitik Jg. 53, Heft 7/8, S.32-42

Dahme, H.-J./F. Hegner u.a. 1980: Die Neuorganisation der ambulanten Sozial- und Gesundheitspflege. Bielefeld: Kleine

Evers, A./Th. Olk 1996: Von der pflegerischen Versorgung zu hilfreichen Arrangements. In: A. Evers/Th. Olk (Hg.) Wohlfahrtspluralismus. Opladen: Westdeutscher Verlag, S.347-372

Fraunhofer-Institut für Arbeitswirtschaft und Organisation 2006: Projektbeschreibung „Pflege 2020“. In: www.pflege2020.de/fhg/Images/ Pflege2020_Projekt-beschreibung_tcm331-73947.pdf (abgerufen am: 15.07.2007)
Hegner, F./U. Lakemann 1989: Familienhaushalt und Erwerbtätigkeit. In: R. Nave-Herz/M. Markefka (Hg.), Handbuch der Familien- und Jugendforschung, Band 1. Neuwied/Frankfurt a.M.: Luchterhand, S. 491-511

Hegner, F. 1991: Angebotsketten und verkettete Organisationsprinzipien der Versorgung. Perspektiven für die Gestaltung der Sozial- und Gesundheitsdienste in West- und Ostdeutschland. In: R.P. Nippert u.a. (Hg.), Kritik und Engagement. Festschrift für Christian von Ferber zum 65. Geburtstag. München: Oldenbourg, S. 225-238

Hegner, F. 1996: Wohlfahrtspluralismus und Wohlfahrtspluralität. In: A Evers/T. Olk (Hg.), Wohlfahrtspluralismus. Opladen: Westdeutscher Verlag, S. 166-185

Hegner, F. 2000: Qualität und Produktivität im Sozial- und Gesundheitsbereich. In: J. König u.a. (Hg.), Qualitätsmanagement und Informationstechnologien im Sozialmarkt. Starnberg: R.S. Schulz, S. 79-94

Kaufmann, F.-X. 1997: Herausforderungen des Sozialstaates. Frankfurt a.M.: Suhrkamp

Kaufmann, F.-X. 2005: Schrumpfende Gesellschaft. Vom Bevölkerungsrückgang und seine Folgen. Frankfurt a.M.: Suhrkamp

Landenberger, M./M. Münch 2001: Innovation in der Pflege. Bern: Huber

Neumeister, H. 1962: Organisierte Menschlichkeit? Grenzen des sozialen Fortschritts. Freiburg i.B.: Herder

\section{Die Neuauflage}

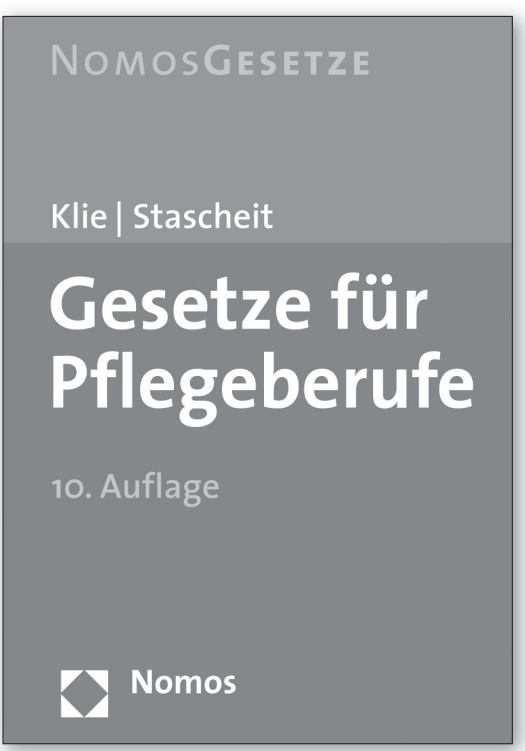

Bitte bestellen Sie bei Ihrer Buchhandlung oder bei Nomos | Telefon 07221/2104-37 | Fax -43 | www.nomos.de | sabine.horn@nomos.de

\section{Gesetze für Pflegeberufe}

Von Prof. Dr. Thomas Klie, FH Freiburg und Prof. Ulrich Stascheit, FH Frankfurt/M.

10. Auflage 2007, 1.204 S., brosch., 19,90 €, ISBN 978-3-8329-2475-1

Vertiefte Rechtskenntnisse im Bereich der ambulanten und stationären Pflege, im Berufs- und Tarifrecht, im Haftungs- und Strafrecht und insbesondere im Recht der Kranken- und Pflegeversicherung gewinnen für Angehörige der Pflegeberufe, Behörden sowie Auszubildende und Studierende der Fachhochschulen zunehmend an Bedeutung.

Die Sammlung fasst die erforderlichen Rechtsgrundlagen jetzt schon in 10. Auflage topaktuell und preiswert zusammen. Berücksichtigt wurden die umfangreichen Änderungen der Sozialgesetzbücher, z.B. durch das KrankenhausentgeltG, das KrankenhausfinanzierungsG, das ArzneimittelG oder die Neuregelungen der BundespflegesatzVO. 